\title{
Sleep Disorder in Tinnitus Patients
}

\author{
Jae-Min Jeon ${ }^{\mathbb{D}}$, Seok-Youl Choi ${ }^{\mathbb{D}}$, Jong-Geun Lee ${ }^{\mathbb{D}}$, Jee Won Moon ${ }^{\mathbb{D}}$, \\ Sung-Won Chae ${ }^{\mathbb{D}}$, and Jae-Jun Song ${ }^{(D)}$ \\ Department of Otorhinolaryngology-Head and Neck Surgery, Korea University College of Medicine, Seoul, Korea
}

\author{
이명 환자에서의 수면장애에 대한 연구 \\ 전재민 · 최석열 · 이종근 · 문지원 · 채성원 · 송재준 \\ 고려대학교 의과대학 이비인후-두경부외과학교실
}

\author{
Received October 5, 2020 \\ Revised January 14, 2021 \\ Accepted January 29, 2021 \\ Address for correspondence \\ Jae-Jun Song, MD, PhD \\ Department of Otorhinolaryngology- \\ Head and Neck Surgery, \\ Guro Hospital, \\ Korea University \\ College of Medicine, \\ 148 Gurodong-ro, Guro-gu, \\ Seoul 08308, Korea \\ Tel +82-2-2626-3191 \\ Fax +82-2-868-0475 \\ E-mail jjsong23@gmail.com
}

Background and Objectives One of the most common complaint of tinnitus patients has been sleep disorder. The aim of this study was to evaluate the relationship between sleep disorder and tinnitus.

Subjects and Method Patients with tinnitus from July 2018 till August 2019 were enrolled. Subjects who had any acute/chronic disease in the inner and middle ear or external auditory canal, sleep apnea and had more than 3 caffeinated beverages a day was excluded. Study participants completed Beck Depression Inventory (BDI), the tinnitus symptom questionnaire, tinnitus handicap inventory (THI), Pittsburg Sleep Quality Inventory (PSQI), pure tone audiometry, a full otologic examination and an interview on past medical history.

Results THI was correlated with PSQI global score and BDI. The PSQI subscale most relevant to THI was PSQI 1 (subjective sleep quality). Hearing impairment was correlated with the THI functional subscale. BDI was individually related to PSQI.

Conclusion Tinnitus is found to be highly related to sleep disorder, suggesting a common pathway of aggravation and treatment target. Tinnitus is also more affected by sleep disorder than by depression. A further examination and treatment of comorbid sleep disorder in tinnitus patients is recommended. Korean J Otorhinolaryngol-Head Neck Surg 2021;64(11):792-9

\section{Introduction}

Tinnitus is defined as recognizing a specific sound without any external stimuli. The Korean National Health Insurance Service uses a total of 27 billion won to treat and examine tinnitus patients with an increase of $2.3 \%$ every year. An analysis of Korean National Health and Nutrition Examination Survey conducted from 2009 to 2011, revealed the prevalence of tinnitus as $9.7 \%$ with a higher percentage in senile populations. ${ }^{1)}$ As the Korean population is rapidly aging, the prevalence and

This is an Open Access article distributed under the terms of the Creative Commons Attribution Non-Commercial License (https://creativecommons.org/licenses/by-nc/4.0) which permits unrestricted non-commercial use, distribution, and reproduction in any medium, provided the original work is properly cited. total medical cost of tinnitus is expected to increase continuously and dramatically.

Although commonly associated with hearing loss, the exact etiology of tinnitus is uncertain. More recently, 'neurophysiological' model of tinnitus has been studied rigorously. As periphery hearing organs receive temporary or permanent damage, a novel pattern of brain activity is generated by the altered connection of central nervous system neurons. This causes excitatory and inhibitory signal imbalance in the auditory and non-auditory cortex, which produce a series of neural activity transmitting between brainstem and cortex in a self-sustaining manner resulting in persistent tinnitus. ${ }^{2)}$

As tinnitus persists, it may cause fatigues, stress, and in- 
somnia and can also manifest as psychological disorders such as depression, anxiety disorder, and cognitive disorder. Previous studies have shown a high prevalence of depression, anxiety and stress in tinnitus patients. ${ }^{3)}$

The most common complaint of tinnitus patients has been sleep disorder and in other studies, the prevalence was as high as $56.9 \%$ and was the most commonly mentioned problem. ${ }^{4}$ Although the exact relationship between sleep disorder and tinnitus has not been elucidated, significant co-morbidity rate and the role of cognitive behavioral therapy (CBT) in both sleep disorder and tinnitus raise interest in the relationship of two diseases. ${ }^{5)}$ However, most of the previous studies that conducted elucidating the relationship of two diseases did not employed a validated measurement tool for sleep disorder.

Thus, in order to elucidate the relationship between sleep disorder and tinnitus we used validated questionnaires specializing in each disease category with an addition of depression questionnaires as they are well-documented correlation between both sleep and tinnitus. ${ }^{6,7)}$ We also acquired additional information such as patients' sex, age, underlying medical condition in order to eliminate confounding factors.

\section{Subjects and Methods}

All participants were patients who visited our otorhinolaryngology clinic between 1 July 2018 and 31 August 2019. The study was approved by the Institutional Review Board (IRB No. 2018GR0322), and it gained informed consents from all the patients. All patients underwent pure tone audiometry (PTA), a full otologic examination with a detailed enquiry of their past medical history. Study was conducted on only those whose chief complaint was chronic idiopathic tinnitus (lasting for more than 3 months) and who were more than 13 years old without and other etiology. Then the patient completed four questionnaires listed below. We also obtained basic demographic information such as sex, age and a detailed interview, and chart review was performed to identify the past medical conditions and history.

Participants who had any suspicion of acute/chronic disease in the inner, middle ear or external auditory canal, sleep apnea, central nervous system disease and who consumed more than 3 cups of caffeinated beverage per day were excluded from the study.

\section{Pittsburg Sleep Quality Inventory (PSQI)}

The Korean version of PSQI was used to evaluate the sleep quality of test subjects. PSQI is currently the most commonly used tool for assessing subjective sleep quality. ${ }^{8,9)}$ It was originally developed and published in 1988 by Pittsburg University and included 7 sub-categories which is equally weighted on a $0-3$ scale. These sub-categories are subjective sleep quality (PSQI 1), sleep latency (PSQI 2), sleep duration (PSQI 3), habitual sleep efficiency (PSQI 4), sleep disturbance (PSQI 5), use of sleep medication (PSQI 6), and daytime dysfunction (PSQI 7). They are added to obtain a global PSQI score which ranges from 0 to 21 . Higher scores indicated worse sleep quality and a global PSQI score greater than 5 yielded a diagnostic sensitivity of $89.6 \%$ and specificity of $86.5 \%$ for sleep disorder. ${ }^{8)}$

\section{Tinnitus handicap inventory (THI)}

THI is the most widely used questionnaire evaluating tinnitus-related disability. The Korean version was developed and validated by $\mathrm{Kim}$, et al. ${ }^{10)}$ in 2002 . The questionnaire consists of 25 questions and for each item, a score of $0,2,4$ is assigned and the total score range is from 0 to 100 . THI is divided into 3 subscales and they are functional subscale (THI-F), emotional subscale (THI-E) and catastrophic subscale (THIC). A total score is acquired by adding all the subscale scores and a higher score indicates a greater handicap from tinnitus and measuring by the total score, 5 categories are used; no handicap (0-16), mild handicap (18-36), moderate handicap (38-56), severe handicap (58-76), and catastrophic handicap $(78-100){ }^{11)}$

\section{Beck Depression Inventory (BDI)}

In order to evaluate depression symptoms, we used a Korean version of BDI, which was translated and validated by Lee and Song ${ }^{12)}$ in 1991. It is a 21-question multiple-choice selfreport inventory and it measures the severity of depression. For each question, the respondent scores it on a scale of 0 to 3 and thus the total score ranges from $0-63$. Higher the total score, the higher to depressive symptoms are and it is divided into 4 categories; minimal depression (0-9), mild depression (10-18), moderate depression (19-29), severe depression $(30-63) .{ }^{12)}$

\section{Tinnitus symptom questionnaire}

We also had a set of questions that asked the specific symptom of tinnitus. The question included, which side the tinnitus was heard, the duration of tinnitus in months, the nature of the onset in tinnitus, the tone of tinnitus, if the tinnitus changed by background noise, if there was any tinnitus inducing situation. 


\section{Hearing assessment}

Hearing status was assessed using PTA and the mean hearing threshold were expressed as the weighted four frequency average of $0.5,1,2,3 \mathrm{kHz}$. Normal hearing was defined as mean threshold less than $26 \mathrm{~dB}$. Hearing threshold of $26-40$ $\mathrm{dB}$ was considered mild, 41-55 dB moderate, 56-70 dB moderate to severe hearing loss, and $71-90 \mathrm{~dB}$ as severe hearing loss.

\section{Statistical analysis}

On data analysis, SPSS software (version 22.0, IBM Corp., Armonk, NY, USA) was used. The data was expressed as

Table 1. Clinical characteristics of tinnitus patients $(n=122)$

\begin{tabular}{|c|c|}
\hline Characteristics & $\mathrm{n}(\%)$ \\
\hline Age & $58.7 \pm 12.9(23-85)$ \\
\hline \multicolumn{2}{|l|}{ Sex } \\
\hline Male & $66(54.1)$ \\
\hline Female & $56(45.9)$ \\
\hline \multicolumn{2}{|l|}{ Localization of tinnitus } \\
\hline Right & $33(27.0)$ \\
\hline Left & $34(27.9)$ \\
\hline Binaural & $55(45.1)$ \\
\hline \multicolumn{2}{|l|}{ Hearing status } \\
\hline Normal & $39(32.0)$ \\
\hline Impairment & $83(68.0)$ \\
\hline PSQI & $9.0 \pm 4.0(1-20)$ \\
\hline PSQI 1 (subjective sleep quality) & $1.4 \pm 0.6(0-3)$ \\
\hline PSQI 2 (sleep latency) & $1.5 \pm 1.2(0-3)$ \\
\hline PSQI 3 (sleep duration) & $2.2 \pm 1.1(0-3)$ \\
\hline PSQI 4 (habitual sleep efficiency) & $1.9 \pm 1.6(0-3)$ \\
\hline PSQI 5 (sleep disturbance) & $1.0 \pm 0.2(0-2)$ \\
\hline PSQI 6 (use of sleep medication) & $0.2 \pm 0.5(0-3)$ \\
\hline PSQI 7 (daytime dysfunction) & $0.6 \pm 0.6(0-3)$ \\
\hline THI & $37.5 \pm 25.4(0-98)$ \\
\hline THI-F & $15.5 \pm 11.5(0-42)$ \\
\hline THI-E & $13.5 \pm 10.3(0-36)$ \\
\hline THI-C & $7.9 \pm 6.0(0-22)$ \\
\hline BDI & $8.8 \pm 7.0(0-35)$ \\
\hline HTN & $28(22.8)$ \\
\hline DM & $7(5.7)$ \\
\hline Ischemic heart disease history & $5(4.1)$ \\
\hline Steroid use & $1(0.8)$ \\
\hline Duration of tinnitus (months) & $49.7 \pm 67.6(3-365)$ \\
\hline Change by noise & $78(63.4)$ \\
\hline Tinnitus inducing situation & $23(18.7)$ \\
\hline
\end{tabular}

Data are presented as mean \pm standard deviation ( $\min -\max$ ). PSQI, Pittsburg Sleep Quality Inventory; THI, tinnitus handicap inventory; THI-F, THI functional subscale; THI-E, THI emotional subscale; THI-C, THI catastrophic subscale; BDI, Beck Depression Inventory; HTN, hypertension; DM, diabetes mellitus mean \pm standard deviation (min-max) for quantitative measures and comparison between two selected groups was done using Student's t-test, $\chi^{2}$ test and Fisher's exact test. Simple linear regression was used to identify the linear relationship between factors. The multiple linear regression model was utilized to evaluate the relationship between sleep disorder and tinnitus handicap scores using stepwise protocol. $p$-values $<0.05$ were accepted as statistically significant results.

\section{Results}

\section{Patient characteristics}

A total of 122 subjects were enrolled for the study, and there was no significant difference in the number of men and women. Sixty seven (54.9\%) subjects had unilateral tinnitus and $55(45.1 \%)$ had bilateral tinnitus. The mean score of PSQI and THI for all subject was $9.0 \pm 4.0$ and $37.5 \pm 25.4$, respectively (Table 1).

\section{Analysis of associated factors in tinnitus}

Simple linear regression analysis looking at THI and PSQI global score revealed a linear relationship between these factors and showed statistical significance $(p<0.05)$ (Fig. 1).

We ran a multiple linear regression analysis using a stepwise protocol to identify the statistical correlation between each factor. Age, sex, hypertension, diabetes mellitus, tinnitus location, tinnitus duration, hearing status of tinnitus side ear, change of tinnitus by noise or situation, BDI, PSQI global score was included in the analysis. The past medical history of steroid use and ischemic heart disease was exempt because of their extreme values with very few cases.

The THI total score was statistically correlated with PSQI

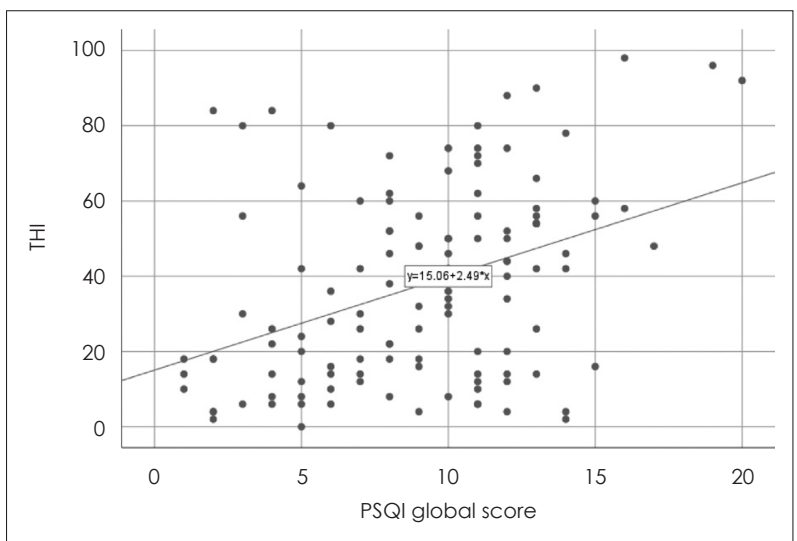

Fig. 1. Simple linear regression analysis of THI and $P S Q I$ global score $(p<0.05)$. THI, tinnitus handicap inventory; PSQI, Pittsburg Sleep Quality Inventory. 
global score and BDI (Table 2). Subscales of THI; functional, emotional, and catastrophic were analyzed separately for a closer look. THI-F was significantly correlated with PSQI global score, BDI and hearing impairment. However, THI-E and THI-C was only statistically relevant with PSQI global score (Table 3).

Further analysis was conducted using PSQI subscale scores. The THI total score was statistically correlated with PSQI 1 (subjective sleep quality), PSQI 7 (daytime dysfunction) and BDI (Table 4). The functional subscale of tinnitus was correlated with PSQI 1 (subjective sleep quality), PSQI 7 (daytime dysfunction), BDI score and hearing impairment. Emotional subscale of tinnitus was correlated with PSQI 1 (subjective sleep quality), PSQI 7 (daytime dysfunction) and BDI. The catastrophic subscale of THI was statistically correlated with PSQI 1 (subjective sleep quality) and PSQI 6 (use of sleep medicine) (Table 4).

\section{Analysis of associated factors in sleep disorder}

Test subjects were divided into two groups according to the PSQI score of 5 to distinguish between sleep disordered subjects and normal subjects. There were 95 (77.9\%) subjects with sleep disorder and 27 (22.1\%) without sleep problems (Table 5).

Table 5 shows a comparison by $\chi^{2}$ test and Fisher's exact test and Student's t-test between subjects with and without sleep

Table 2. Relationship between PSQI global score and THI

\begin{tabular}{lcccc}
\hline & $B$ & $B$ & $S E$ & $p$ value \\
\hline PSQI global score & 1.926 & 0.304 & 0.593 & 0.002 \\
BDI & 0.689 & 0.191 & 0.337 & 0.043 \\
\hline
\end{tabular}

PSQI, Pittsburg Sleep Quality Inventory; THI, tinnitus handicap inventory; B, regression coefficient; $B$, standardized beta coefficient; SE, standard error; BDI, Beck Depression Inventory

Table 3. Relationship between PSQI global score and THI subscale

\begin{tabular}{lccc}
\hline & B & SE & P value \\
\hline THI-F & & & \\
$\quad$ PSQI global score & 0.725 & 0.269 & 0.008 \\
BDI & 0.348 & 0.153 & 0.025 \\
$\quad$ Hearing impairment & 4.356 & 2.051 & 0.036 \\
$\begin{array}{l}\text { THI-E } \\
\quad \text { PSQI global score }\end{array}$ & 0.906 & 0.220 & $<0.001$ \\
$\begin{array}{l}\text { THI-C } \\
\quad \text { PSQI global score }\end{array}$ & 0.504 & 0.130 & $<0.001$ \\
\hline
\end{tabular}

PSQI, Pittsburg Sleep Quality Inventory; THI, tinnitus handicap inventory; B, regression coefficient; SE, standard error; THI-F, THI functional subscale; BDI, Beck Depression Inventory; THI-E, THI emotional subscale; THI-C, THI catastrophic subscale disorder. PSQI score and patients who reported tinnitus that changed by background noise was significantly different in two groups. Other conditions did not affect the outcome.

\section{Discussion}

Insomnia is the most frequent complaints associated with tinnitus, and there have been many previous studies trying to elucidate its relationship. In a recent study by Jia, et al., ${ }^{13)} 263$ tinnitus patients and control group underwent Pittsburg Sleep Quality Index, Hamilton Anxiety Scale, Hamilton Depression Scale and PTA. There was significantly more subjects with sleep disorder in tinnitus group, and accompanying anxiety and depression were also important factors affecting sleep quality. A questionnaire study by Miguel, et al., ${ }^{14)}$ examined 85 tinnitus patients and 32 tinnitus patients that was also diagnosed insomnia. The patients underwent Tinnitus Reaction Questionnaire and Insomnia Severity Index. It showed a robust correlation between the Tinnitus Reaction Questionnaire and Insomnia Severity Index. The Tinnitus Reaction Questionnaire (especially the emotional sub-score) showed a good predictive value of sleep disturbance. A retrospective analysis of 182 patients in 2015 with chronic tinnitus who completed the Tinnitus Questionnaire and the Regensburg Insomnia Scale, also showed higher prevalence of insomnia

Table 4. Relationship between PSQI sub-score and total THI/THI subscale

\begin{tabular}{lccc}
\hline & B & SE & p value \\
\hline THI & & & \\
PSQI 1 (subjective sleep quality) & 7.861 & 2.791 & 0.006 \\
PSQI 7 (daytime dysfunction) & 5.835 & 2.649 & 0.030 \\
BDI & 0.807 & 0.314 & 0.011 \\
THI-F & & & \\
PSQI 1 (subjective sleep quality) & 3.102 & 1.238 & 0.014 \\
PSQI 7 (daytime dysfunction) & 3.398 & 1.176 & 0.005 \\
BDI & 0.372 & 0.140 & 0.009 \\
Hearing impairment & 5.088 & 1.969 & 0.011 \\
THI-E & & & \\
$\quad$ PSQI 1 (subjective sleep quality) & 2.479 & 1.174 & 0.037 \\
PSQI 7 (daytime dysfunction) & 2.261 & 1.115 & 0.045 \\
BDI & 0.294 & 0.132 & 0.028 \\
THI-C & & & \\
$\quad$ PSQI 1 (subjective sleep quality) & 1.919 & 0.664 & 0.005 \\
PSQI 6 (use of sleep medicine) & 4.623 & 0.769 & 0.037 \\
\hline
\end{tabular}

PSQI, Pittsburg Sleep Quality Inventory; THI, tinnitus handicap inventory; B, Regression coefficient; SE, standard error; BDI, Beck Depression Inventory; THI-F, THI functional subscale; THI-E, THI emotional subscale; THI-C, THI catastrophic subscale 
Table 5. Predictors of sleep disorder in tinnitus patients $(n=122)$

\begin{tabular}{|c|c|c|c|}
\hline Characteristics & $P S Q I \leq 5(n=27,22.1 \%)$ & PSQI > $5(n=95,77.9 \%)$ & $p$ value \\
\hline Age & $57.1 \pm 12.1(28-82)$ & $59.1 \pm 13.1(23-85)$ & 0.473 \\
\hline Sex & & & 0.055 \\
\hline Male & $19(70.4)$ & $47(49.5)$ & \\
\hline Female & $8(29.6)$ & $48(50.5)$ & \\
\hline BDI & $4.4 \pm 4.5(0-19)$ & $10.1 \pm 7.1(0-35)$ & $<0.001$ \\
\hline HTN & $3(11.1)$ & $25(26.3)$ & 0.097 \\
\hline DM & $1(3.7)$ & $6(6.3)$ & 0.607 \\
\hline Steroid use & $1(3.7)$ & $0(0)$ & 0.221 \\
\hline Ischemic heart disease history & $0(0)$ & $5(5.3)$ & 0.580 \\
\hline Localization of tinnitus & & & 0.458 \\
\hline Right & $7(25.9)$ & $26(27.4)$ & \\
\hline Left & $10(37.0)$ & $24(25.3)$ & \\
\hline Binaural & $10(37.0)$ & $45(47.4)$ & \\
\hline Duration of tinnitus (months) & $39.8 \pm 46.7(3-180)$ & $52.5 \pm 72.7(3-365)$ & 0.392 \\
\hline Hearing status of tinnitus side & & & 0.396 \\
\hline Normal & $10(37.0)$ & $29(30.5)$ & \\
\hline Mild & $10(37.0)$ & $43(45.3)$ & \\
\hline Moderate & $4(14.8)$ & $18(18.9)$ & \\
\hline Moderate-severe & $3(11.1)$ & $3(3.2)$ & \\
\hline Severe & $0(0)$ & $2(2.1)$ & \\
\hline Change by noise & $22(81.5)$ & $56(58.9)$ & 0.030 \\
\hline Tinnitus inducing situation & $3(11.1)$ & $20(21.1)$ & 0.244 \\
\hline
\end{tabular}

Data are presented as n (\%). PSQI, Pittsburg Sleep Quality Inventory; BDI, Beck Depression Inventory; HTN, hypertension; DM, diabetes mellitus

in tinnitus patients and tinnitus distress correlated with sleep disturbance. ${ }^{15)}$ While as the previous studies only analyzed correlation between the two disease entities, our statistical model (multivariate linear regression model) revealed a possible linear and causal relationship between insomnia and tinnitus, and included depression as a confounding factor. Furthermore, we performed subanalysis based on the subscales of THI and PSQI.

PSQI global score was significantly correlated with THI and its all subscales according to the Tables 2 and 3. In addition, PSQI 1 (subjective sleep quality) correlated strongly with the total THI and all its subscales (Table 4). Therefore, it is possible to infer that insomnia might not merely be a co-morbid disease but a disease sharing a course of aggravation with tinnitus. Indeed, the Tinnitus Reaction Questionnaire and the mean Insomnia Severity Index scores were significantly higher in a group of tinnitus with insomnia compared to the group of tinnitus only according to the study conducted by Miguel, et al. ${ }^{14)}$ In addition, study by Folmer and Griest ${ }^{16)}$ showed that the Tinnitus Severity Index score increased significantly as the degree of subjective tinnitus interference with sleep got worse. Our results showed similar results as well (Fig. 1).
Published in 2006, the neurophysiological model of tinnitus emphasized the limbic system in pathological mechanism of tinnitus. ${ }^{17)}$ Tinnitus is different from other natural sounds in the aspect of subjective sensations it causes. Patients report negative emotions such as confusion and anxiety. The negative subjective emotions influence the limbic system in a stressful manner that can amplify the subcortical auditory system reaction resulting in a vicious cycle. Another system affected by the limbic system is the autonomic nervous system, and it is influenced by the cerebral cortex and the limbic system through feedback mechanisms, thereby indirectly affects patients' psychology. An activated autonomic nervous system can trigger a flight or fight mode with pupil dilation, heartbeat increase, tensing muscle and decreased sleep. Hyperarousal caused by autonomic nervous system is one of the mechanisms related to the insomnia. There is also an increasing body of evidence that hyperarousal in animal models for tinnitus and insomnia shows the similarities in the activation pattern of limbic and autonomous brain region further supporting our assumption. ${ }^{18)}$

Looking more closely at other PSQI subscales that correlated with THI, PSQI 6 (use of sleep medication) had statistically 
significant association with THI-C only while PSQI 7 (daytime dysfunction) with total THI, THI-F, and THI-E. It is possible interpretation that the patients suffering from severe tinnitus have difficulty falling asleep and tend to feel catastrophic about their tinnitus simultaneously, thereby resorting to sleep medication. However, PSQI 2, 3, 4, and 5 were not statistically associated with THI scores, which gave contrary results to the study conducted in 2015 that performed meta-analysis of PSQI in terms of its diagnostic validity. It concluded that each of the subscales sufficiently represented each domain of sleep quality in global score and was useful in detecting attributes of poor sleep quality for targeted investigation and treatment. However, as there has been no stable optimal cut off score for the subscale and factors affecting structure of sleep quality has not been constant between and within groups, this interpretation of statistical analysis may not be clinically meaningful. ${ }^{19)}$ More studies on tinnitus patients with PSQI subscales are needed to obtain more reliable interpretation of these results.

The effort of finding an effective treatment for tinnitus is still in progress. The recently published European guideline for tinnitus only had strong recommendation for cognitive behavioral therapy (CBT), and the most of the commonly utilized treatments currently lacked consistent evidence. ${ }^{20)}$ CBT approaches tinnitus on its psychological distress and resulting problem. It also hypothesizes that tinnitus and its distress originates from misguided information processing, behavior habits and emotional reactivity. CBT is also strongly recommended for treatment of insomnia. It is a well-studied method of treatment and it has a model of specialized treatment called CBT for insomnia (CBTI). A recent meta-analysis study proved its effectiveness in insomnia. ${ }^{21)}$ Another noteworthy study was published in 2019 applying CBTI to tinnitus patients with insomnia and it showed reduced tinnitus distress after a six-week intervention. ${ }^{22)}$ Overall, these studies suggest a strong relationship between insomnia and tinnitus, and it is worth applying CBT to tinnitus patients with insomnia because these diseases share a course of aggravation.

To our knowledge, this is one of the few studies analyzing depression and sleep disorder in tinnitus patients with validated tools. Most of the studies on sleep disorder and tinnitus did not incorporate depression as confounding factor and its interaction with sleep disorder. For an instance, an analysis of American National Health Survey of 2007 revealed a strong relationship between tinnitus, anxiety, depression and sleep disorder. However, this analysis was performed via limited evaluation tools such as duration of sleep or single question ask- ing if they had anxiety, depression or tinnitus. ${ }^{6)}$ In our study, BDI was correlated with total THI total score and THI-F, and the standardized beta coefficient of BDI was considerably smaller than that of PSQI global score. However, there have been multiple studies proving the stronger correlation between tinnitus and depression. For example, a study by Trevis, et al. ${ }^{23)}$ used THI, BDI, State Trait Anxiety Inventory and Obsessive Beliefs Questionnaire and found a strong relationship between tinnitus and depression mediated by anxiety. Study conducted by Kehrle, et al., ${ }^{24)}$ looking at normal hearing individuals with pure tone threshold of $<25 \mathrm{~dB}$ with THI and BDI also found strong correlation. A national health survey analysis in 2016 revealed a close association of anxiety, depression, and greater workdays missed. However, the analysis itself was based on the national survey relying on single questions such as how much concern of tinnitus or anxiety causes the subject and how many hours of sleep they get every night. ${ }^{6}$ The different degree of correlation among studies might be due to the difference in study population and the specific method of assessing each disease's severity. However, the above studies have not considered sleep disorder as confounding factor, and a correlation of sleep disorder to depression has been well documented in the past studies. ${ }^{7)}$ Sine the depression and sleep disorders are individually correlated, the previous studies without correction of confounding factor may have overestimated its effect size of depression on tinnitus. A similar conclusion was reached by a study conducted by Aazh and Moore, ${ }^{25)}$ and his study used Hospital Anxiety and Depression Scale with THI and Insomnia Severity Index. This study concluded that although the depression was predicted by THI, most of its effects were mediated by insomnia, anxiety and hyperacusis. From our study and previous studies, it can be inferred that the sleep disorder may have a more profound effect on tinnitus than depression.

It is well documented from large scale health surveys that tinnitus prevalence is closely related to hearing loss, aging, ototoxicity and noise exposure history. ${ }^{26)}$ In our study, presence of hearing impairment only affected THI-F in both PSQI global score analysis and PSQI subscale analysis. It can be interpreted as hearing impairment having a more direct impact on the functional side of tinnitus. In terms of THI total score, THI-E and THI-C; although the relationship between hearing loss and tinnitus is clear, its impact is variable according to the source and study group. There are studies showing patients without any hearing impairment suffering from tinnitus ranging from $20 \%$ as high as $40 \%{ }^{26)}$ The same study with 
a different population set may reveal a stronger or weaker correlation.

Age was not correlated with tinnitus handicap in our study. This is probably due to the relatively small sample size as nationwide studies have revealed a strong relationship. ${ }^{1,26)}$ There was a lack of participants that had a history of prescribed ototoxic drugs to analyze in a meaningful way. We did not include question about noise exposure history in our study. This is since although it does affect prevalence of tinnitus, it is unmodifiable in treatment terms and we wanted to focus more on sleep disorder and tinnitus.

The following study differs from previous studies as it was only recruited from tinnitus patients. This recruitment more closely mimics clinical setting of an otolaryngologists and thus a more practical description of the tinnitus patient's characteristics. For clinicians it may be important to actively identify tinnitus patients also with sleep disorders as our results convey a more casual relationship rather than a mere co-morbid correlation. In practical terms, actively prescribing CBT to these patients may be beneficial. Although a recent European guideline's strong recommendation for CBT on treating tinnitus, in Korea there is an absolute lack of availability. This is due to its relative novel approach and restriction of public medical insurance policy and governance. Prescribing CBT for comorbid insomnia is more feasible and may prove to be very effective as it is the recommended treatment for both disease.

However, the study is not without limitations. First, it is prone to sample bias. All test subjects were recruited in tertiary medical center and thus it is possible that patients with higher disease burden were recruited; this result may not represent the general population. Second, it is a single-center study. The size of the study population had its limitation and a larger size population study will be more accurate. Third, it has a lack of control group that did not suffer from tinnitus. A further multicentered large-scale study is needed for a more complete picture.

In conclusion, PSQI global score and its subscales were significantly correlated to the total THI score and its subscales, indicating a strong association between tinnitus and sleep disorder and thereby, suggesting a common treatment target. In our study, sleep disorder and depression were closely associated each other in tinnitus patients, and sleep disorder had more effect on aggravation of tinnitus than depression based on the standardized beta coefficients of PSQI global score and BDI score. A further examination and treatment of comorbid sleep disorder in tinnitus patients is recommended.

\section{Acknowledgments}

None.

\section{Author Contribution}

Conceptualization: Jae-Min Jeon, Sung-Won Chae, Jae-Jun Song. Data curation: Seok-Youl Choi, Jong-Geun Lee, Jee Won Moon. Formal analysis: Jae-Min Jeon, Seok-Youl Choi. Methodology: Sung-Won Chae, Jae-Jun Song. Project administration: Jae-Min Jeon, Jong-Geun Lee, Jee Won Moon. Visualization: Jae-Min Jeon. Writing — original draft: Jae-Min Jeon. Writing — review \& editing: Jae-Min Jeon, Jae-Jun Song.

\section{ORCIDs}

Jae-Jun Song

Jae-Min Jeon

Seok-Youl Choi

Jong-Geun Lee

Jee Won Moon

Sung-Won Chae

https://orcid.org/0000-0002-8488-9091 https://orcid.org/0000-0002-7900-1991 https://orcid.org/0000-0001-9280-6308 https://orcid.org/0000-0002-8138-969X https://orcid.org/0000-0003-2307-1824 https://orcid.org/0000-0001-6401-352X

\section{REFERENCES}

1) Park KH, Lee SH, Koo JW, Park HY, Lee KY, Choi YS, et al. Prevalence and associated factors of tinnitus: Data from the Korean National Health and Nutrition Examination Survey 2009-2011. J Epidemiol 2014;24(5):417-26.

2) Saunders JC. The role of central nervous system plasticity in tinnitus. J Commun Disord 2007;40(4):313-34.

3) Gomaa MAM, Elmagd MHA, Elbadry MM, Kader RMA. Depression, anxiety and stress scale in patients with tinnitus and hearing loss. Eur Arch Otorhinolaryngol 2014;271(8):2177-84.

4) Tyler RS, Baker LJ. Difficulties experienced by tinnitus sufferers. J Speech Hear Disord 1983;48(2):150-4.

5) McKenna L, Handscomb L, Hoare DJ, Hall DA. A scientific cognitive-behavioral model of tinnitus: Novel conceptualizations of tinnitus distress. Front Neurol 2014;5:196.

6) Bhatt JM, Bhattacharyya N, Lin HW. Relationships between tinnitus and the prevalence of anxiety and depression. Laryngoscope 2017; 127(2):466-9.

7) Murphy MJ, Peterson MJ. Sleep disturbances in depression. Sleep Med Clin 2015;10(1):17-23.

8) Buysse DJ, Reynolds CF 3rd, Monk TH, Berman SR, Kupfer DJ. The Pittsburgh Sleep Quality Index: A new instrument for psychiatric practice and research. Psychiatry Res 1989;28(2):193-213.

9) Sohn SI, Kim DH, Lee MY, Cho YW. The reliability and validity of the Korean version of the Pittsburgh Sleep Quality Index. Sleep Breath 2012;16(3):803-12.

10) Kim JH, Lee SY, Kim CH, Lim SL, Shin J, Chung WH, et al. Reliability and validity of a Korean adaptation of the tinnitus handicap inventory. Korean J Otolaryngol 2002;45(4):328-34.

11) McCombe A, Baguley D, Coles R, McKenna L, McKinney C, Windle-Taylor P, et al. Guidelines for the grading of tinnitus severity: The results of a working group commissioned by the British Association of Otolaryngologists, Head and Neck Surgeons, 1999. Clin Otolaryngol Allied Sci 2001;26(5):388-93.

12) Lee Y, Song YS. A study of the reliability and the validity of the BDI, SDS, and MMPI-D scales. Korean J Clin Psychol 1991;10(1): 98-113.

13) Jia R, Liu B, Cheng L, Sun FX. Clinical research on sleep quality in patients with tinnitus. Lin Chung Er Bi Yan Hou Tou Jing Wai Ke Za Zhi 2019;33(10):961-5.

14) Miguel GS, Yaremchuk K, Roth T, Peterson E. The effect of insomnia 
on tinnitus. Ann Otol Rhinol Laryngol 2014;123(10):696-700.

15) Schecklmann M, Pregler M, Kreuzer PM, Poeppl TB, Lehner A, Crönlein T, et al. Psychophysiological associations between chronic tinnitus and sleep: A cross validation of tinnitus and insomnia questionnaires. Biomed Res Int 2015;2015:461090.

16) Folmer RL, Griest SE. Tinnitus and insomnia. Am J Otolaryngol 2000;21(5):287-93.

17) Jastreboff PJ, Jastreboff MM. Tinnitus retraining therapy: A different view on tinnitus. ORL J Otorhinolaryngol Relat Spec 2006;68(1): 23-9; discussion 29-30.

18) Wallhäusser-Franke E, Schredl M, Delb W. Tinnitus and insomnia: Is hyperarousal the common denominator? Sleep Med Rev 2013; 17(1):65-74

19) Mollayeva T, Thurairajah P, Burton K, Mollayeva S, Shapiro CM, Colantonio A. The Pittsburgh Sleep Quality Index as a screening tool for sleep dysfunction in clinical and non-clinical samples: A systematic review and meta-analysis. Sleep Med Rev 2016;25:5273.

20) Cima RFF, Mazurek B, Haider H, Kikidis D, Lapira A, Noreña A, et al. A multidisciplinary European guideline for tinnitus: Diagnostics, assessment, and treatment. HNO 2019;67(Suppl 1):10-42.

21) van Straten A, van der Zweerde T, Kleiboer A, Cuijpers P, Morin $\mathrm{CM}$, Lancee J. Cognitive and behavioral therapies in the treatment of insomnia: A meta-analysis. Sleep Med Rev 2018;38:3-16.

22) Marks E, McKenna L, Vogt F. Cognitive behavioural therapy for tinnitus-related insomnia: Evaluating a new treatment approach. Int J Audiol 2019;58(5):311-6.

23) Trevis KJ, McLachlan NM, Wilson SJ. Psychological mediators of chronic tinnitus: The critical role of depression. J Affect Disord 2016;204:234-40.

24) Kehrle HM, Sampaio ALL, Granjeiro RC, de Oliveira TS, Oliveira CACP. Tinnitus annoyance in normal-hearing individuals: Correlation with depression and anxiety. Ann Otol Rhinol Laryngol 2016;125(3):185-94.

25) Aazh H, Moore BCJ. Factors associated with depression in patients with tinnitus and hyperacusis. Am J Audiol 2017;26(4):562-9.

26) Shargorodsky J, Curhan GC, Farwell WR. Prevalence and characteristics of tinnitus among US adults. Am J Med 2010; 123(8):711-8. 